\title{
Face mask use among individuals who are not medically diagnosed with COVID-19: A lack of evidence for and against and implications around public health recommendations.
}

Keshini Madara Marasinghe ( $D$ kmmarasi@uwaterloo.ca )

University of Waterloo https://orcid.org/0000-0001-6201-860X

Systematic Review

Keywords: COVID-19, mask, public health, communicable disease

Posted Date: July 17th, 2020

DOl: https://doi.org/10.21203/rs.3.rs-16701/v4

License: (9) This work is licensed under a Creative Commons Attribution 4.0 International License. Read Full License

Version of Record: A version of this preprint was published on September 2nd, 2020. See the published version at https://doi.org/10.14202/IJOH.2020.109-117. 


\section{Abstract}

\section{Introduction}

Since the beginning of the COVID-19 outbreak, public health professionals have been constantly making decisions on face mask use among individuals who are not medically diagnosed with COVID-19 or "healthy individuals" to limit the spread of COVID-19. While some countries have strongly recommended face masks for "healthy individuals", other countries have recommended against it. Public health recommendations that have been provided to this population since the beginning of the outbreak have been controversial, contradicting, and inconsistent around the world. The purpose of this paper is to understand available evidence around the effectiveness or ineffectiveness of face mask use in limiting the spread of COVID-19 among individuals who have not yet been diagnosed with COVID-19 and most importantly, to understand the state of knowledge that the public health recommendations that have been provided since the beginning of the COVID-19 outbreak are based on.

\section{Methods}

A systematic review was conducted to identify studies that investigated the use of face masks to limit the spread of COVID-19 among "healthy individuals".

\section{Results}

No studies were found, demonstrating a lack of evidence for and against face mask use suggesting implications around public health recommendations provided to "healthy individuals" since the beginning of the COVID-19 outbreak.

\section{Conclusions}

Three and a half months into the COVID-19 outbreak (December $2019-2^{\text {nd }}$ week of April 2020), there are no peer-reviewed scientific studies that have investigated the effectiveness or ineffectiveness of face mask use among "healthy individuals". Yet, very strong public health recommendations have been provided on whether they should or should not wear face masks to limit the spread of COVID-19. A lack of scientific evidence heavily questions the basis of these public health recommendations provided at a very early, yet a crucial stage of an outbreak. This finding and a further look at public health recommendations conclude that there is a clear need for more concentrated research around face mask use among healthy individuals as well as public health recommendations that are evidence-based; precautionary in the absence of evidence; based on benefit-risk assessment; transparent; and globally aligned in order to provide the most successful guidelines during an infectious disease outbreak.

\section{Background}

Since the beginning of the COVID-19 outbreak, public health professionals around the world have been constantly making decisions on the use of face masks among individuals 
who are not medically diagnosed with Coronavirus disease 2019 (COVID-19) ${ }^{1}$. While public health professionals in some countries have strongly recommended the use of face masks, other countries have recommended not to use face masks as a precautionary method to limit the transmission of COVID-19 among "healthy individuals". Public health recommendations that have been provided to this population since the beginning of the outbreak have been controversial, contradicting and inconsistent around the world. Due to contradicting recommendations, there is confusion and lack of clarity around the use and effectiveness of face masks in limiting the spread of COVID-19 among this specific population. The purpose of this paper is to understand the available evidence around the effectiveness of face mask use in limiting the spread of COVID-19 among individuals who have not yet been diagnosed with COVID-19 and to understand the state of knowledge that the public health recommendations that have been provided since the beginning of the COVID-19 outbreak are based on.

Medical or surgical masks ${ }^{2}$ or N95 respirators, ${ }^{3}$ hereinafter referred to as face masks have been widely used by healthy individuals in various parts of the world such as in Asia for years as a precautionary method to limit the spread of infectious diseases. During COVID19 outbreak, countries such as Australia, Canada and the United States of America recommended against the use of face masks for "healthy people" or individuals who are not medically diagnosed with COVID-19, while health officials in countries like China, Indonesia, and the Philippines supported the use of face masks among healthy individuals as a precautionary method to limit the spread of COVID-19. [1] [2] [3] [4] [5] [6] [7] [8]

It is important to highlight the population of focus of this paper - individuals who are not medically diagnosed with COVID-19, also generally have been referred to as "healthy individuals" by public health professionals and the media. It is important to note that just because these individuals have not yet been diagnosed with COVID-19, it does not mean that they are healthy. Individuals who are not medically diagnosed with COVID-19 include those who may have been exposed to the virus and are asymptomatic; pre-symptomatic; symptomatic but have not yet been diagnosed with COVID-19 and therefore not aware that they have COVID-19 and; those who have not been exposed to the virus. The first three groups of individuals are impossible to distinguish from one another in terms of whether they carry COVID-19 and a risk of spreading the virus in public spaces. Not recommending face masks for this population assuming that they are all healthy is problematic. Provided below are some examples that illustrate the variations in public health recommendations on face mask use that have been provided by public health professionals to this specific population since the beginning of the COVID-19 outbreak. 


\begin{tabular}{|c|c|}
\hline The Source/Date & Recommendation \\
\hline $\begin{array}{l}\text { The Philippines, } \\
\text { former secretary } \\
\text { of health, } \\
31 \text { Tan } 2020\end{array}$ & $\begin{array}{l}\text { "It [surgical mask] is not } 100 \text { percent effective because there are still gaps where } \\
\text { air can flow through, but it's better than not wearing any. It's about } 90 \text { percent } \\
\text { effective. It may be safe not to wear face masks in areas where one is sure that no } \\
\text { person with coronavirus has entered. If you are at the mall, you don't know who } \\
\text { else are there if they came from China and has the coronavirus." [5] }\end{array}$ \\
\hline $\begin{array}{l}\text { Indonesia, the } \\
\text { secretary health } \\
\text { ministry of } \\
\text { disease control } \\
\text { and } \\
\text { environmental } \\
\text { health } \\
\text { directorate } \\
\text { general, }\end{array}$ & $\begin{array}{l}\text { "As long as we use it correctly, a [surgical] mask is enough protection from the } \\
\text { virus or bacteria. The masks should be used mainly by sick people to prevent the } \\
\text { spread of the virus while coughing. The mask also protects healthy people from } \\
\text { being infected while in public places." [8] }\end{array}$ \\
\hline
\end{tabular}

11 Feb. 2020

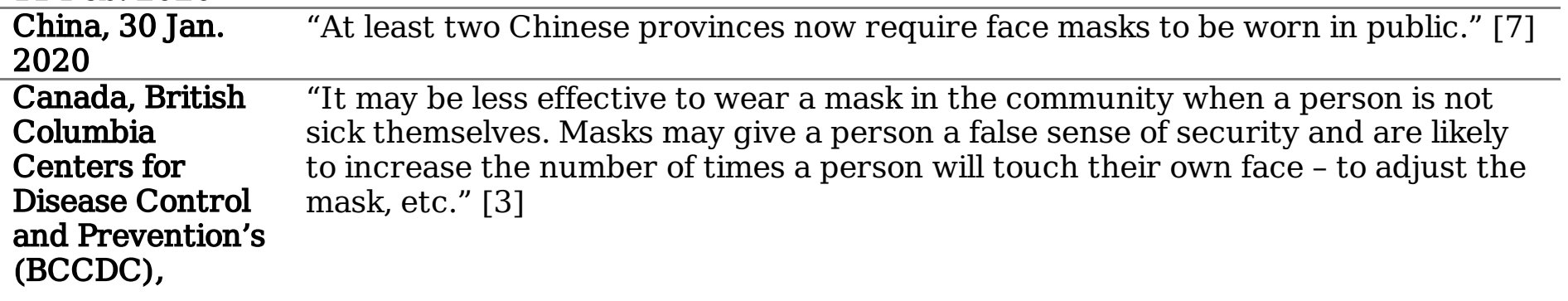

30 Jan. 2020

Canada, chief “'We never recommend wearing a mask in public' because many people don't use medical officer of them properly: Reaching underneath them to touch your face, for instance, Ontario, spreads germs. N95 respirator masks (so named because they're designed to stop 95 percent of small particles from reaching the nose and mouth) only work if they

20 Jan. 2020 fit properly, which they won't for children or people with facial hair." [1]
Canada, Immunology Professor at the University of Toronto, 23 Jan. 2020
The USA, The Center for the National Center for Immunization and Respiratory Diseases, 02 Mar. 2020 Australia, The state of New South Wales, "The issue with [the surgical mask] is it obviously doesn't protect your eyes and they're loosely fitting and there's an opportunity for something to come within areas where it's not tightly fitted, through your nose or neck. The coronavirus is transmitted through human-to-human contact, which includes droplets produced while sneezing or coughing. Wearing a surgical mask, that is very well-fitted, would reduce the amount of virus you'd be exposed to." [9]
"'We don't routinely recommend the use of face masks by the public to prevent respiratory illness. And we certainly are not recommending that at this time for this new virus. We want our actions to be evidence-based and appropriate to the current circumstance' which did not justify the use of face masks for people who have not been directly exposed to the virus". [2]
"Face masks are not recommended for the general population." [4]

13 Feb. 2020

$\begin{array}{ll}\text { The World } & \text { "Wearing a medical mask is one of the prevention measures to limit the spread of } \\ \text { Health } & \text { certain respiratory diseases including the Novel Coronavirus (2019-nCoV) in } \\ \text { Organization } & \text { affected areas. For healthy people wear a mask only if you are taking care of a } \\ \text { (WHO), } & \text { person with suspected 2019-nCoV infection", where the definition of "healthy } \\ \text { 29 January, 2020 } & \text { people" is unclear. [11] }\end{array}$


Table 1: Varying public health recommendations from public health professionals on face mask use for individuals who are not medically diagnosed with COVID-19.

As demonstrated in Table 1, public health officials in countries like China, Indonesia, and the Philippines are in support of face mask use to limit the spread of COVID-19 among those who are not medically diagnosed with COVID-19, whereas countries like Australia, Canada, the United States of America and the WHO provide a mixture of recommendations, but mostly not in support for face mask use for individuals who are not medically diagnosed with COVID-19. Due to contradicting public health recommendations provided during the COVID-19 outbreak on face mask use among healthy individuals, it has become clear that there is a need to further study and reconstruct better approaches to providing noncontradictory, consistent, and successful public health recommendations that are applicable to all kinds of populations during a public health crisis.

\section{Methods}

A systematic review was carried out to search databases Cochrane Library (1993 - $2^{\text {nd }}$ week of April 2020), EMBASE (1974 - $2^{\text {nd }}$ week of April 2020), Google Scholar (2004 $-2^{\text {nd }}$ week of April 2020), PubMed (1950 - $2^{\text {nd }}$ week of April 2020) and Scopus (1966 - $2^{\text {nd }}$ week of April 2020) were searched for relevant studies. Two groups of keywords were combined: those relating to COVID-19 and face masks (Figure 1). Retrieved articles were searched for relevant articles by screening the title and abstract by one reviewer.

\section{Inclusion criteria}

1) Randomized control trials (RCTs), cohort, retrospective or prospective studies that evaluated the effectiveness or ineffectiveness of face masks in limiting the spread of COVID-19 among the general population or in community settings.

2) Studies that were in English.

\section{Exclusion criteria}

If the abstract did not relate to the effectiveness or ineffectiveness of face masks in limiting the spread of COVID-19 among the general population or in community settings, the study was excluded. Commentaries were excluded.

\section{Results}

No peer-reviewed scientific studies were found that investigated the effectiveness or ineffectiveness of face mask use among individuals who are not medically diagnosed with COVID-19 resulting in an empty 
review (Figure 2). Yet, very strong public health recommendations have been provided on whether "healthy individuals" should or should not wear face masks as a preventive measure to limit the spread of COVID-19 since the beginning of the COVID-19 outbreak. This finding, which is a lack of scientific evidence for and against face mask use, heavily questions the basis of public health recommendations that have been provided to the public at an early stage of an outbreak, where preventive measures are most important.

\section{Discussion}

COVID-19 is still (as of April 2020) a fairly new virus therefore, the result of an empty review is not surprising. However, the public health recommendations that have been provided in the absence of evidence at a very early stage of an outbreak (December 2019 - April 2020) are questionable. The findings of this study highlight the lack of evidence around the effectiveness and ineffectiveness of face mask use and sheds light on how this finding affect public health recommendations. COVID-19 pandemic has revealed that there is a need for public health professionals and decision makers to better understand and practise approaches that are mutually agreed upon in order to provide the most successful recommendations during a global crisis. Understanding and practising more effective approaches to providing public health recommendations is crucial to avoid inconsistencies and contradictions in public health recommendations especially during an infectious disease outbreak. Conflicts and deeper concerns around the public health recommendations that have been provided since the beginning of the COVID-19 outbreak are discussed below followed by effective approaches to providing successful public health recommendations during an infectious disease outbreak.

In a situation where an individual who has not yet been medically diagnosed with COVID-19 (asymptomatic, pre-symptomatic or symptomatic but not yet diagnosed), who is not wearing a face mask as per public health recommendations, coughs or sneezes without covering their face, they can be releasing respiratory droplets, according to the WHO. [10] The WHO states that "people can also catch COVID-19 if they breathe in droplets from a person with COVID-19 who coughs out or exhales droplets. This is why it is important to stay more than 1 meter (3 feet) away from a person who is sick." [10] If these individuals are not wearing face masks as per public health recommendations, the risk of spreading the infection through coughing or sneezing out droplets is higher. At the same time, if healthy individuals in close proximity are also not wearing face masks because of advice against the use of face masks, which has been the case in many countries since the start of the outbreak, they would be at a higher risk of contracting COVID-19 through respiratory droplets. [10] The WHO has provided a recommendation stating that "relatives or caregivers to individuals with suspected 2019-nCoV infection with mild respiratory symptoms should wear a medical mask when in the same room with the affected individual", suggesting that wearing a medical mask is useful at least to some extent in protecting those who are healthy. [11] This recommendation has likely been provided to err on the side of caution, which is appreciated. However, it is questionable why the same recommendation does not apply to healthy 
individuals who can be at an equal risk of exposure to an infected individual (asymptomatic, presymptomatic, symptomatic but not yet diagnosed) in public spaces. If face masks are effective in protecting a healthy family member from a sick family member by keeping out droplets at home, it is contradicting to suggest that the same face masks are ineffective in limiting transmission in public spaces. Does wearing a face mask protect healthy individuals in close proximity in public spaces at least to some extent? Is not wearing a face mask at all in public spaces beneficial than wearing one? These are the kind of questions that create confusion and controversy. Therefore, it is imperative that public health professionals consider and thoroughly study these questions prior to providing strong recommendations. There are few approaches to providing successful recommendations during challenging times and they are discussed below.

\section{Evidence-based public health recommendations}

Public health researchers suggest that decision makers should always integrate scientific evidence when planning and implementing programs, developing policies and evaluating progress. [12] [13] Practising evidence-based approaches in public health increases the availability of higher quality information, the likelihood of successful prevention programs and policies, and efficiency in the use of resources. [12] [14] [15] [16] [17] Recommendations that are not supported by scientific evidence can create confusion and controversy and also increase the risk of unnecessary spread of the infection as illustrated in the above example. However, while evidence-based recommendations are ideal, it is imperative that public health professionals recognize the best approaches to take when there is a lack of evidence around a precautionary method. During an infectious disease outbreak, it is unrealistic and unsuccessful to wait until the presence of evidence to provide evidence-based recommendations. In the absence of

evidence, precautionary recommendations should be given greater consideration in order to prevent and lessen the widespread of the outbreak.

\section{Precautionary recommendations}

The lack of evidence on the effectiveness of face mask use in limiting the spread of COVID-19 has been one of the main justifications for countries to have recommended against face mask use among healthy individuals at the initial stages of the outbreak. The WHO suggests, "the precautionary principle states that in the case of serious or irreversible threats to the health of humans or the ecosystem, acknowledged scientific uncertainty should not be used as a reason to postpone preventive measures." [18] Many Western countries refused to follow the precautionary principle on providing recommendations around face mask use until there was immense public criticism. Canada for example recommended against face mask use for "healthy individuals" until April 2020 solely because of a lack of scientific evidence. Even though there is minimal evidence to support the use of face masks as one of the precautionary methods in limiting the spread of COVID-19, many Asian countries such as the Philippines have recommended its 
use widely as a precautionary practice very early on in the COVID-19 pandemic, which is an example of following the precautionary principle in order to provide precautionary recommendations as also advised by the WHO. [5]

In the example discussed above under "discussion", there is no known effective approach to prevent transmission in crowded spaces other than to assume that individuals who are not wearing face masks practise other precautionary methods such as hand hygiene and respiratory etiquette. Rather than relying on such assumptions, it is better to err on the side of caution and provide precautionary public health recommendations such as the recommendation to wear face masks in public because if the assumptions do not hold true, then the risk of spread increases in the absence of precautionary recommendations.

Such increased risk that can be reduced by precautionary recommendations is problematic especially for vulnerable populations and healthy individuals in the community who are often around these vulnerable populations such as family members of immunocompromised individuals. People with pre-existing conditions such as cancer, diabetes, heart disease and renal or chronic lung disease are more vulnerable to becoming severely ill with COVID-19. [19] [20] Their healthy family members being at a higher risk, puts these vulnerable individuals at a higher risk of being exposed to the virus. Even with minimal evidence, having precautionary recommendations in place can be more beneficial than waiting for evidence to provide perfect public health recommendations during an outbreak.

Benefits versus risks

Another justification for providing recommendations against face mask use has been the risks associated with face mask use. Potential risks of face mask use as identified by public health and health professionals are: increased possibility of spreading germs due to the misuse of face masks such as touching the outside of the face masks and retouching underneath the face mask to touch the face; increased likelihood of people touching their own faces because of the face masks, for example, to adjust the masks; establishment of a false sense of security among those who wear face masks; and ineffectiveness due to unfitting of face masks especially among children and those with facial hair. [1] [2] [3] [9] [11] [21] Public health and health professionals must question if the potential risks associated with wearing face masks incorrectly outweigh the potential benefits of wearing face masks correctly when followed by other precautionary practices. In the case where potential benefits of wearing face masks appropriately cannot be outweighed against the risks of wearing face masks, taking actions to eliminate potential risks associated with wearing face masks through health promotion and health education initiatives may be a safer approach rather than recommending the public not to wear a face mask at all because of the risks associated with incorrect face mask use. These potential risks can be minimized by educating the public about the risks and how to correctly use face masks to avoid the risks. 


\section{Transparency}

Another point to consider when providing public health recommendations during a public health emergency is transparency. During an infectious disease outbreak, there might be delays in obtaining treatments and executing interventions and resources may be limited. [22] Public recommendations and guidelines, therefore, is the most important tool available to public health professionals in managing a risk. [22] Transparency during a health emergency is vital as information plays an important role in maintaining core public health objectives. [22] Transparency in public health recommendations provides the right information needed to survive the emergency. [22] [23] [24] Public health scholars state that "transparency is a necessary, if not sufficient, condition for accountable decision-making and for the promotion of public trust." [22] Transparency about the unknown and the known are both equally important to build and promote public trust in order to achieve public adherence to recommendations. [22]

In the case of the COVID-19 outbreak, whether recommendations are provided in the presence or absence of evidence must be transparent to the public. Where there is evidence transparency, the public is more likely to understand what scientific evidence the recommendations are based on and therefore, be more confident in adhering to the recommendations. For example, in the case of medical professionals' request to halt the hoarding of face masks among the public, merely saying "masks do not help" has not been helpful in preventing the public from continuing to buy face masks. [25] [26] With transparency around the available evidence of their ineffectiveness, public health and health professionals can be confident and consistent in recommending the public not to buy face masks and as a result, avoid situations such as running out of face masks for healthcare workers.

Similarly, when recommending against face mask use due to concerns around the false sense of security that face masks may bring, knowledge transparency becomes important. [2] [3] [11] A false sense of security is the feeling of being safer than one really is. [27] In the context of COVID-19 and face mask use, a false sense of security means that the individuals may feel that they are safe from contracting COVID19 because they are wearing face masks while that may not be the case. In the case of such concern, the public must be educated about the "false sense of security" that wearing a face mask may bring to individuals and how it may contribute to the increased risks associated with wearing face masks or neglecting other precautionary actions.

All of this information and concerns must be effectively communicated with the public through reliable media to be as transparent as possible. In order to achieve the goals behind the recommendations, it is imperative to be transparent with the public about both current evidence and knowledge public health recommendations are based on. 


\section{Global alignment on public health recommendations}

The last but not least important point to consider when providing public health recommendations during an outbreak is that public health recommendations must align globally. Varying recommendations can have a negative effect on countries around the world, especially in developing countries. In some developing countries, there is a severe lack of dissemination of credible information to the public as well as a concerning lack of knowledge among the general population to be able to identify and distinguish credible information from noncredible information. For example, a consulting physician in Sri Lanka says that, "containing the panic and dissemination of misinformation has proved tougher than fighting the actual disease [COVID-19]." [28] [29] In these populations, most people tend to trust what is typically seen on social media or other online platforms, especially if the recommendations are coming from developed countries due to the assumption that the practices are evidence-based and well thought out because they are coming from more advanced and developed countries.

In the case of face mask use, when populations in developing countries observe on online platforms a wide use of face masks to limit the spread of COVID-19 or recommendations advising the use of face masks, they may be likely to follow these recommendations without questioning the evidence behind the practices or recommendations. Influenced by what they see on online platforms, populations in these countries could take away the wrong message that merely wearing face masks will protect them from contracting COVID-19. Similarly, if there are recommendations to not wear face masks, those recommendations might also be followed without taking into consideration other advice that should follow along such as practising hand hygiene and respiratory hygiene.

In developing countries with less advanced health care systems, confusion due to varying recommendations can lead to having a worse negative impact on their population as well as healthcare systems. Considering these factors, global alignment on public health recommendations and decisions among public health professionals and entities is crucial when proving recommendations amid an outbreak such as COVID-19.

\section{Future research on face mask use}

Further investigation into the effectiveness of face mask use among healthy individuals is important and timely for a number of reasons. If currently available face masks are not effective in limiting the spread of COVID-19 among healthy individuals or have significant limitations to its effectiveness, with advanced technology and innovations, further research can investigate novel approaches to invent and improve the 
efficiency of face masks to limit the transmission of COVID-19. While investigating the effectiveness of face masks will be beneficial in limiting the spread of COVID-19, further research can open doors to investigating, understanding, and designing more advanced personal protective equipment (PPE) for situations where PPE may be required in community settings. Additional research can help identify and distinguish between the types of face masks that are best for different populations, and address possible stigma and existing cultural differences associated with face mask use. Moreover, information about the effectiveness of face masks will provide health professionals insight into whether there is a need to advise global manufacturers to increase the production of face masks, which addresses growing concerns such as face mask hoarding by the public, limited availability of face masks for healthcare workers, and the need for mandatory control over supply and price of face masks through legislation in countries that recommend the use of face masks to limit the spread of COVID-19. [30] [31] [32]

This research is important, especially today as there is an immense use of face masks around the world despite the uncertainty around its effectiveness and contradicting public health recommendations. In the case of a pandemic, knowing the effectiveness of face masks ahead of time of the crisis would be extremely useful. While research surrounding the biology, epidemiology and behaviour of the new virus is extremely important and a priority at the moment, studying every method of prevention is equally important as it will not only provide the public with knowledge and instructions that they can rely on and confidently practise, but also save lives and reduce suffering and the expenditure of healthcare resources. Further investigation into precautionary methods will also provide the adequate knowledge and confidence for public health professionals to provide recommendations that are evidence-based; precautionary in the absence of evidence; based on benefit-risk assessment; transparent; and globally aligned.

\section{Conclusions}

The result of an empty review questions the basis of public health recommendations that have been provided to the public at a very early, yet a crucial stage of an outbreak. However, this finding reveals that there is a need to better understand how to provide non-contradictory, consistent and successful public health recommendations during a global health crisis. While there is a clear need for more concentrated research around face mask use among healthy individuals, this paper calls for closer attention to be given to constructing public health recommendations that are evidence-based; precautionary in the absence of evidence; based on benefit-risk assessment; transparent; and globally aligned in order to provide the most successful guidelines during an infectious disease outbreak.

\section{List Of Abbreviations}

British Columbia Centers for Disease Control and Prevention (BC CDC) 
Cable News Network (CNN)

Centers for Disease Control and Prevention (CDC)

Coronavirus disease 2019 (COVID-19)

Food and Drug Administration (FDA)

Middle East respiratory syndrome coronavirus (MERS-CoV)

Novel Coronavirus (2019-nCoV)

Randomized control trials (RCTs)

World Health Organization (WHO)

\section{Footnotes}

${ }^{1}$ Individuals who are "not medically diagnosed with COVID-19" include those who may have been exposed to the virus and are asymptomatic; pre-symptomatic; symptomatic but have not yet been diagnosed with COVID-19 by a healthcare professional therefore may not be aware that they have COVID19 and; those who have not been exposed to the virus.

2United States, Centers for Disease Control and Prevention (CDC) describes a surgical mask as a face mask that provides barrier protection against large particle droplets that can be released when a wearer talks, coughs, or sneezes. [33] CDC notes that medical or surgical masks do not effectively filter inhaled small particles, fumes, or vapors. [33] They are "primarily used to protect patients and healthcare workers from people who may have a respiratory infection" according to CDC. [33]

${ }^{3}$ The Food and Drug Administration (FDA) of the United States, describes N95 respirators as masks that are designed to "achieve a very close facial fit and very efficient filtration of airborne particles." [34] N95 respirators block at least 95 percent of very small (0.3 micron) test particles minimizing the wearers' respiratory exposure to airborne infectious agents. [34] [35] FDA also states that if N95 masks are properly fitted, the filtration capabilities of N95 respirators surpass those of surgical masks. [34] However, even a properly fitted N95 respirator does not completely eliminate the risk of illness or death according to FDA. [34]

\section{Declarations}


Ethics and dissemination: Not applicable.

\section{Authors' contributions}

Keshini Madara Marasinghe led and conducted the review and wrote the rest of the paper.

Funding: This research received no specific grant from any funding agency in the public, commercial or not-for-profit sectors.

Data Sharing: Data sharing not applicable as no datasets generated and/or analysed for this study.

Competing interests: The author declares that they have no competing interests.

Patient and Public Involvement statement

Patients or the public WERE NOT involved in the design, or conduct, or reporting, or dissemination plans of our research

Acknowledgements

The author would like to thank the following individuals for their professional advice and feedback, technical input, and review of this literature review.

Dr. Jostacio Moreno Lapitan, WHO

Loïc Garçon, WHO

Bridget Lee, MPH

\section{References}


[1] What can I do about the Wuhan coronavirus? A guide for Canadians of what's helpful, and what's not. The Globe and Mail. 2020. https://www.theglobeandmail.com/canada/articlewuhan-novel-coronavirus-2019ncov-canada-health-explainer/. Accessed 04 February 2020.

[2] CDC says Americans shouldn't wear face masks to prevent coronavirus - here are 3 other reasons not to wear them Market Watch. 2020. https://www.marketwatch.com/story/the-cdcsays-americans-dont-have-to-wear-facemasks-because-of-coronavirus-2020-01-30. Accessed 06 February 2020.

[3] Little S. What the BC CDC says you're getting wrong about the novel coronavirus. Global News. 2020. https://globalnews.ca/news/6486026/bc-cdc-coronavirus-misconceptions/. Accessed 05 February 2020.

[4] Alerts - Novel coronavirus - Frequently asked questions. 2020.

https://www.health.nsw.gov.au/Infectious/alerts/Pages/coronavirus-faqs.aspx. Accessed 13 February 2020.

[5] Lopez ML. Are cloth masks effective as protection from the novel coronavirus? CNN Philippines. 2020. https://www.cnnphilippines.com/news/2020/1/31/Cloth-masks-protectionnovel-coronavirus.html. Accessed 03 February 2020.

[6] Shen M, Peng Z, Xiao Y, Zhang L. Modelling the epidemic trend of the 2019 novel coronavirus outbreak in China. bioRxiv. 2020.

[7] Wernau J, Xie SY. Coronavirus fears drive demand for face masks, but some experts doubt them. 2020. https://www.wsj.com/articles/chinese-authorities-promote-face-masks-but-someexperts-question-them-11580422149. Accessed 03 February 2020.

[8] N95 masks are overkill against coronavirus, Health Ministry official suggests. The Jakarta Post. 2020. https://www.thejakartapost.com/news/2020/02/11/n95-masks-are-overkillagainst-coronavirus-health-ministry-official-suggests.html. Accessed 13 February 2020.

[9] Abedi M. Demand for face masks on the rise amid coronavirus outbreak - but are they effective? Global News. 2020. https://globalnews.ca/news/6451929/coronavirus-china-facemasks/. Accessed 03 February 2020.

[10] World Health Organization, Q\&A on coronaviruses (COVID-19). 2020.

https://www.who.int/news-room/q-a-detail/q-a-coronaviruses. Accessed 28 February 2020.

[11] World Health Organization. Advice on the use of masks the community, during home care and in health care settings in the context of the novel coronavirus (2019-nCoV) outbreak. 2020. https://www.who.int/publications-detail/advice-on-the-use-of-masks-in-the-community-duringhome-care-and-in-healthcare-settings-in-the-context-of-the-novel-coronavirus-(2019-ncov)outbreak. Accessed 03 February 2020.

[12] Brownson RC, Fielding JE, Maylahn CM, Evidence-Based Public Health: A fundamental concept for public health practice. Annu Rev Public Health. 2009;30:175-201.

[13] McGinnis JM, Does proof matter? Why strong evidence sometimes yields weak action. Am J Health Promot. 2001;15:391-96.

[14] Lhachimi SK, Bala MM, Vanagas G. Evidence-Based Public Health. BioMed Research International. 2016;2016:1-2.

[15] Brownson RC, Baker EA, Leet TL, Gillespie KN. Evidence-Based Public Health. New York: Oxford University Press. 2003.

[16] Hausman AJ. Implications of evidence-based practice for community health. Am J Community Psychol. 2002;30:453-67 
[17] Kohatsu ND, Melton RJ. A health department perspective on the guide to community Preventive Services. Am J Prev Med. 2000:27; 417-21.

[18] World Health Organization, "The precautionary principle: Public health, protection of children and sustainability," 2020. [Online]. Available:

https://www.who.int/hia/examples/overview/whohia076/en/. [Accessed 15 April 2020].

[19] The 2019 Novel Coronavirus (COVID-19). https://www.ontario.ca/page/2019-novelcoronavirus. Accessed 25 January 2020.

[20] World Health Organization. Q\&A on coronaviruses. 2020 https://www.who.int/news-room/q-adetail/q-a-coronaviruses. Accessed 11 February 2020.

[21] Jones AM. Can face masks protect you from the coronavirus? Experts weigh in. CTV News, 2020. https://www.ctvnews.ca/health/can-face-masks-protect-you-from-the-coronavirusexperts-weigh-in-1.4786014. Accessed 03 February 2020.

[22] O'Malley P, Rainford J, Thompson A. Transparency during public health emergencies: from rhetoric to reality. Bull World Health Organ. 2009;87:614-618.

[23] Daniels N. Accountability for reasonableness. BMJ. 2000;2321:1300-1.

[24] Thompson A, Faith K, Gibson J, Upshur R. Pandemic influenza preparedness: an ethical framework to guide decision-making. BMC Med Ethics. 2006;7:E9.

[25] Brown D, Merali F. Toronto doctor sounds alarm about medical mask shortage amid fears of COVID-19. CBC News. https://www.cbc.ca/news/canada/toronto/shortage-medical-maskstoronto-covid-19-1.5480982. Accessed 29 February 2020.

[26] John G. 'Seriously people': Surgeon general urges public to 'stop buying masks' for coronavirus. NBC News. https://www.msn.com/en-us/news/us/seriously-people-surgeongeneral-urges-public-to-stop-buying-masks-for-coronavirus/ar-BB10zmxO. Accessed 29 February 2020.

[27] False Sense Of Security. https://www.merriam-webster.com/dictionary/false sense of security. Accessed 13 February 2020.

[28] Borham M. Containing panic and misinformation tougher than fighting coronavirus. Sunday Observer. 2020. http://www.sundayobserver.Ik/2020/02/02/news-features/'containing-panicand-misinformation-tougher-fighting-coronavirus'. Accessed 23 February 2020.

[29] Khurana D. Misinformation rampant on social media over coronavirus. 2020. https://www.ha-asia.com/misinformation-rampant-on-social-media-over-coronavirus/. Accessed 23 February 2020.

[30] Time for fresh thinking on face mask supply. HKEJ Editorial. 2020. http://www.ejinsight.com/20200213-time-for-fresh-thinking-on-face-mask-supply/. Accessed 13 February 2020.

[31] Coronavirus: Prices of face masks soaring, supplies scarce - WHO. Aljazeera. 2020. https://www.aljazeera.com/ajimpact/coronavirus-prices-face-masks-soaring-supplies-scarce200207220551214.html. Accessed 09 February 2020.

[32] Boseley S. WHO warns of global shortage of face masks and protective suits. The Guardian. 2020. https://www.theguardian.com/world/2020/feb/07/who-warns-global-shortage-facemasks-protective-suits-coronavirus. Accessed 09 February 2020.

[33] CDC. Use of respirators and surgical masks for protection against healthcare hazards - HSPS - 
NIOSH workplace safety and health topic. 2018.

https://www.cdc.gov/niosh/topics/healthcarehsps/respiratory.html. Accessed 07 February 2020.

[34] Center for Devices and Radiological Health. Masks and N95 respirators. 2020.

https://www.fda.gov/medical-devices/personal-protective-equipment-infection-control/masksand-n95-respirators. Accessed 07 February 2020.

[35] Canadian Centre for Occupational Health. Respiratory protection against airborne infectious agents for health care workers. 2020.

https://www.ccohs.ca/oshanswers/prevention/respiratory_protection.html. Accessed 18

February 2020.

Marasinghe, K. M. (2020). Face mask use among individuals who are not medically diagnosed with COVID-19: A lack of evidence for and against and implications around public health

[36]

recommendations. doi: 10.21203/rs.3.rs-16701/v3

\section{Figures}

EMBASE (1974 - $2^{\text {nd }}$ week of April 2020) and Cochrane Library (1993 - $2^{\text {nd }}$ week of April): face piece.mp. or exp protective equipment/ OR exp mask/ or exp surgical mask/ or exp face mask/ or Respirator*.mp. or N95.mp. or Respiratory Protective Devices.mp. or Medical Mask.mp. AND 2019-nCoV.mp. or 2019-novel coronavirus.mp. or 2019 novel coronavirus.mp. or covid-19.mp

Google Scholar (2004 - 2 ${ }^{\text {nd }}$ week of April 2020): "Face mask" OR mask* OR "Facepiece" OR Respirator* OR N95 OR "Respiratory Protective Devices" OR "Medical Mask" OR "surgical mask" AND "2019-nCoV" OR "2019-novel coronavirus" OR "2019 novel coronavirus" OR "COVID-2019"

PubMed (1950 - $2^{\text {nd }}$ week of April 2020): ((((((((((((Face mask[Title/Abstract]) OR mask[Title/Abstract]) OR Face piece[Title/Abstract]) OR respirator[Title/Abstract]) OR N95[Title/Abstract]) OR "Respiratory Protective Devices"[Title/Abstract]) OR "Medical Mask"[Title/Abstract]) OR "surgical mask"[Title/Abstract]) AND "2019-nCoV"[Title/Abstract]) OR "2019-novel coronavirus"[Title/Abstract]) OR "Wuhan coronavirus" [Title/Abstract]) OR "Wuhan seafood market pneumonia virus"[Title/Abstract]) OR "2019 novel coronavirus"[Title/Abstract]) OR "COVID-2019"[Title/Abstract]

Scopus (1966 - 2 nd week of April 2020): (TITLE-ABS-KEY ( "Face mask" OR mask* OR "Facepiece" OR Respirator* OR N95 OR "Respiratory Protective Devices" OR "Medical Mask" OR "surgical mask") AND TITLE-ABS-KEY ("2019nCoV" OR "2019-novel coronavirus" OR "2019 novel coronavirus" OR "COVID-19"))

\section{Figure 1}

Database search terms. 


\section{Databases searched}

Cochrane Library (1993-2 ${ }^{\text {nd }}$ week of Apr 2020)

EMBASE (1974-2 ${ }^{\text {nd }}$ week of Apr 2020)

Google Scholar (2004 - 2 ${ }^{\text {nd }}$ week of Apr 2020)

PubMed (1950-2 $2^{\text {nd }}$ week of Apr 2020)

Scopus (1966-2 ${ }^{\text {nd }}$ week of Apr 2020)

\section{Search Terms}

"Face mask*" OR mask OR masks OR "Face piece*" OR respirator* OR N95 OR "Respiratory Protective Devices" OR "medical mask" OR "surgical mask" OR "Facepiece" OR N95 AND "2019-nCoV" OR "2019novel coronavirus" OR "2019 novel coronavirus" OR "Wuhan coronavirus" OR "Wuhan seafood market pneumonia virus" OR "COVID-19"

\section{Duplicates excluded $=8$}
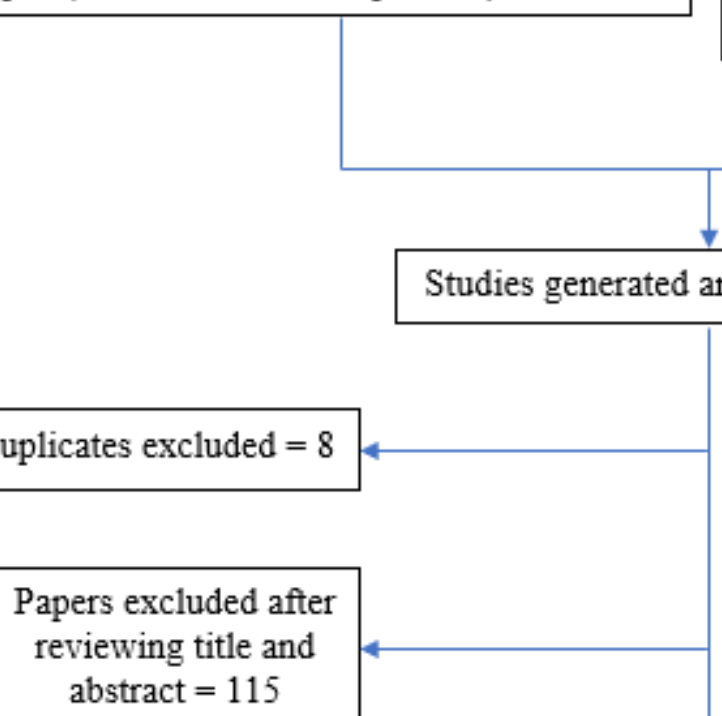

Studies generated and screened $=123$

Figure 2

Schematic diagram of the literature search.

\section{Supplementary Files}

This is a list of supplementary files associated with this preprint. Click to download.

- PRISMAChecklist.docx 